\title{
TEM Study of Lizardite-1T Particles in Toluene Solvent
}

\author{
J. Yan, ${ }^{*}$ X.Z. Li, ${ }^{* *}$ J.Y. Jiang, ${ }^{*}$ L. Tan ${ }^{* * *}$ \\ * Department of Engineering Mechanics, University of Nebraska, Lincoln, NE 68588 \\ ${ }^{* *}$ Nebraska Center for Materials and Nanoscience, University of Nebraska, Lincoln, NE 68588
}

To investigate the self-assembled growth of organosilane molecules carrying $\mathrm{Si}, \mathrm{C}$ and $\mathrm{H}$ to form multiple layers [1], samples were prepared in toluene and then transferred to TEM Cu grids coated with thin $\mathrm{C}$ films for TEM observation. It is surprising that micrometer sized flakes with a main composition of $\mathrm{Mg}$ and $\mathrm{Si}$ (or $\mathrm{Al}$ and $\mathrm{Si}$ ) were occasionally observed in the TEM specimens, later were identified as lizardite-1T phase. To trace down the source of the inorganic particles, all procedures in the sample preparation were carefully checked. The particles of the lizardite-1T phase were confirmed from the organic solvent of toluene.

Figure 1 shows the morphologies of typical particles which are in flake form with irregular shapes, (a) particle size about $8 \mu \mathrm{m}$ and (b) particle size about $6 \mu \mathrm{m}$. The thin areas of the edges of the flakes were used for SAED experiment. EDS analysis reveals that ratio of $\mathrm{Mg}$ and $\mathrm{Si}$ (or $\mathrm{Al}$ and $\mathrm{Si}$ ) is various in different areas of the samples. Figure 2 shows the two zone-axis SAED patterns selected from the experimental tilting series, (a) in hexagonal symmetry, (b) a SAED pattern with tilted angle $14^{\circ}$ away from (a) while keeping one column of reflections unchanged, as marked with arrows. After calibration of the camera constant with the SAED patterns of polycrystalline Au film, the lattice parameter of the hexagonal lattice is derived as $a=5.32 \AA$ from figure 2 (a).

The phase identification was achieved by comparing experimental and simulated diffraction patterns. In combination of the results from both the EDS and SAED experiments, a list of compounds containing $\mathrm{Mg}$ and $\mathrm{Si}$ with hexagonal/trigonal lattice and lattice parameter is about 5.32 $\AA$ were selected from an inorganic crystal database [5]. The structure for the particles is most likely to be the lizardite phase. However, there are two polytypes, lizardite-1T (P31m, $a=5.325(5) \AA$ and $c=7.259(7) \AA)$ and lizardite-2H1 (P6 ${ }_{3} \mathrm{~cm}, a=5.318(4) \AA$ and $\left.c=14.541(7) \AA\right)$. Figure 3 shows the simulated electron diffraction patterns using the structure of the lizardite-1T phase, (a) [001] zone axis and (c) [113] zone axis including first-order Laue zone. The angle between the [001] and [113] is $13.8^{\circ}$. The diffraction intensities were calculated under kinematical diffraction theory with software written by Li [6]. It is obvious that there is a similarity between the intensity distribution of reflection spots in the experimental and simulated diffraction patterns. Simulated patterns using lizardite-2H1 structure were also checked out, the [001] zone axis pattern was found matching to figure 2(a) but there is no pattern matching to figure 2(b) at an angle near $14^{\circ}$ from the [001] zone axis. Thus it has been confirmed that the samples under examination is the lizardite-1T phase. Structural deterioration was observed when the lizardite-1T particles were exposed to the relative higher dose of electron beam.

The results of the experiments should raise cautions for research involving layered assembly. However, it is never our intention to extend this conclusion to other cases, where the amount of such a mineral is yet trivial comparing to major products.

This work was partially supported by the Army Research Office (W911NF-08-1-0190) and NSF CMMI (0825905). 


\section{References}

[1] A. Shimojima, C.W. Wu and K. Kuroda, J. Mater. Chem. 17(2007) 658.

[2] J.C. Rucklidge and J. Zussman, Acta Cryst. 19 (1965) 381.

[3] M. Mellini and P.F. Zanazzi, Am. Mineral. 72 (1987) 943.

[4] A.P. Zhukhlistov and B.B. Zvyagin, Cryst. Report 43 (1998) 950.

[5] ICSD, Inorganic Crystal Structure Database (2008).

[6] X.Z. Li, J. Appl. Cryst. 36 (2003) 956.

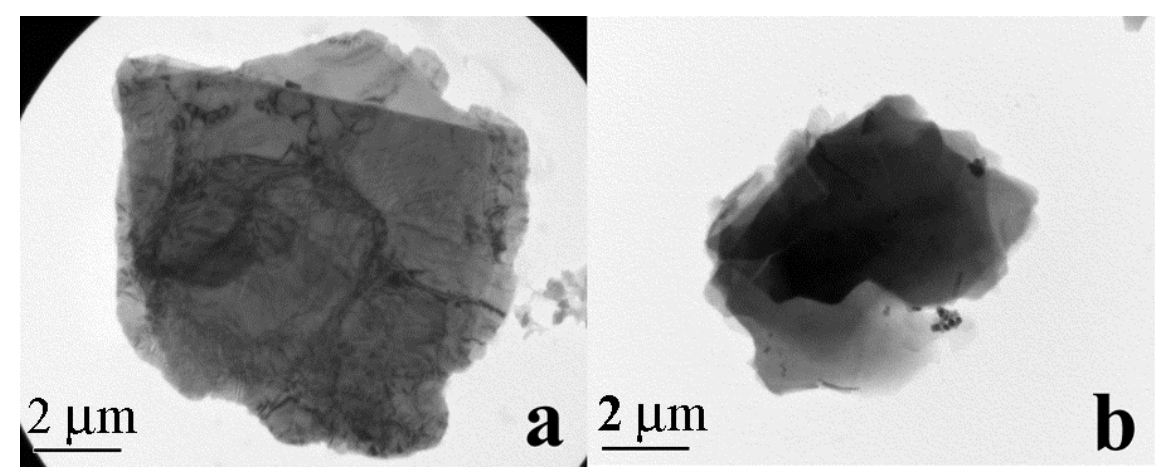

Figure 1. Morphologies of the typical particles with sizes about (a) $8 \mu \mathrm{m}$ and (b) $6 \mu \mathrm{m}$.

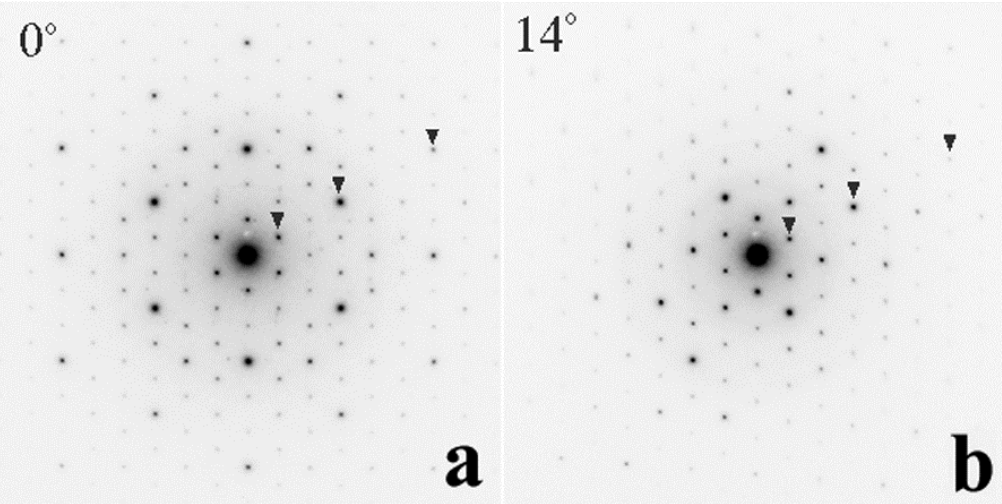

Figure 2. Zone-axis SAED patterns selected from an experimental tilting series.

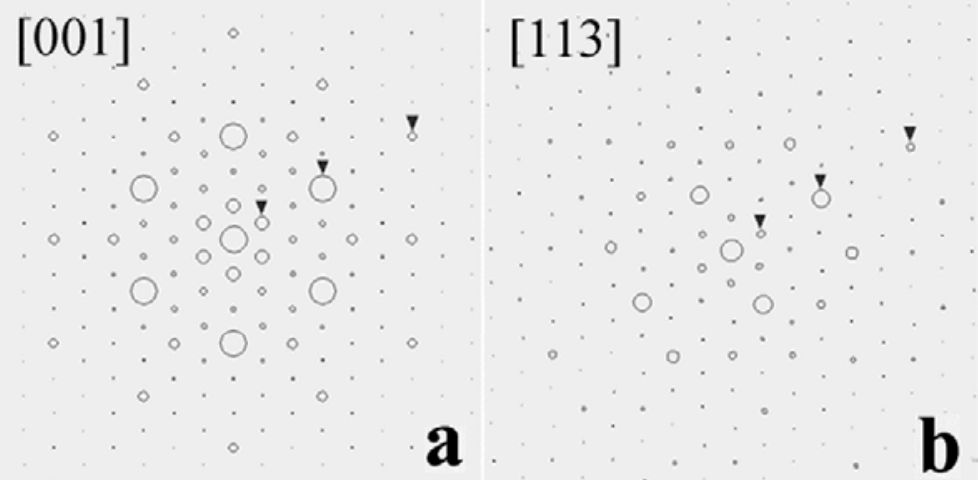

Figure 3. Simulated ED patterns of the lizardite-1T phase, (a) [001] zone axis and (c) [113] zone axis including first-order Laue zone, which correspond to Fig. 2 (a) and (b), respectively. 